\title{
SELEÇÃO DE ANOS-PADRÃO PARA ANÁLISE RÍTMICA EM ESTUDOS DE CONFORTO TÉRMICO: UMA PROPOSTA DE "CONFORTOGRAMAS" A PARTIR DE ÍNDICES
}

\author{
SILVA, Liliane Flávia Guimarães da - lilianeg@ifto.edu.br \\ Doutoranda em Ciências Ambientais - Coordenação de Construção Civil/IFTO \\ SOUZA, Lucas Barbosa e - Ibsgeo@uft.edu.br \\ Doutor em Geografia - Departamento de Geografia/UFT
}

\begin{abstract}
RESUMO: A técnica de análise rítmica aplica-se a diversos estudos climáticos, incluindo estudos em conforto térmico. A presente pesquisa tem como objetivo propor um método de seleção dos anos-padrão para análise rítmica, voltado a estudos em conforto térmico, a partir de um índice e priorizando a característica absoluta do dado, por meio de análise de frequências. A proposta resulta em "Confortogramas", instrumentos utilizados pelo pesquisador para classificar os anos-padrão em habitual, confortável, desconfortável para o frio e desconfortável para o calor. O procedimento proposto é exemplificado numa aplicação prática, na cidade de Palmas, Tocantins. A metodologia apresentou-se eficiente, possibilitando realizar um recorte temporal para análise rítmica que considere o ritmo do comportamento do conforto térmico.
\end{abstract}

Palavras- chaves: Análise rítmica, Índices de Conforto Térmico, Discomfort Index, Palmas (TO)

SELECTION OF STANDARD-YEARS FOR RHYTHMIC ANALYSIS IN THERMAL COMFORT RESEARCH: A PROPOSAL OF "COMFORT DIAGRAMS" FROM INDICES

\begin{abstract}
The technique of rhythmic analysis may be applied to several climatic studies, including thermal comfort researches. The present paper aims to propose a method of selecting the standard-years for rhythmic analysis, dedicated to studies on thermal comfort from an index and prioritizing the absolute characteristic of the data, through frequency analysis. The proposal results in the "Comfort diagrams", which are instruments used by the researcher to classify the standard-years into habitual, comfortable, uncomfortable for the cold and uncomfortable for the heat. The proposed procedure exemplified in a practical application, in the city of Palmas, state of Tocantins, Brazil. The methodology was efficient, making it possible to develop a time selection for rhythmic analysis that considers the rhythm of the thermal comfort behavior.
\end{abstract}

Keywords: Rhythmic analysis, Thermal Comfort Indices, Discomfort Index, Palmas (TO).

\section{INTRODUÇÃO}

A técnica de análise rítmica foi proposta pelo geógrafo brasileiro Carlos Augusto de Figueiredo Monteiro (MONTEIRO, 1971) ${ }^{1}$, a partir de proposta conceitual do geógrafo francês Maximilian Sorre (SORRE, 1951), em que o clima é compreendido como sucessão habitual dos estados atmosféricos, em contraposição ao conceito de clima como o estado médio da atmosfera. A técnica proposta por Monteiro (1971) interpreta o clima por um ritmo de atuação, com destaque para seus aspectos genéticos e dinâmicos, conferindo

\footnotetext{
${ }^{1}$ Destaca-se aqui essa obra de 1971, em que o autor sintetiza e descreve didaticamente a técnica da análise rítmica, mas a construção do conceito foi desenvolvida ao longo de sua vida acadêmica (MONTEIRO $1951 ; 1962 ; 1963 ; 1964 ; 1969)$. Mais informações podem ser consultadas em Monteiro (2015).
} 
uma identidade geográfica à análise climatológica. Essa identidade é conferida pela vinculação do ritmo e tempo (duração), com a sensibilidade e as atividades humanas, configurando a "realidade viva", um dos princípios nas definições climáticas de Sorre (1951).

Para aplicar a técnica de análise rítmica, é necessário verificar a influência dos sistemas atmosféricos atuantes em determinada localidade por meio da "representação concomitante dos elementos fundamentais do clima em unidades de tempo cronológico pelo menos diárias" (MONTEIRO, 1971, p. 9), analisados simultaneamente. Por vezes, em episódios menores, é possível sua análise horária, ou inferior, medido em minutos (RIBEIRO, 1993). Tal mensuração é definida pelo pesquisador a depender do foco da pesquisa.

Diferente da análise estatística, que considera medidas de tendência central e de dispersão, frequente na meteorologia, a análise de Monteiro (1971) prioriza dados brutos. Devido a essa característica, que dificulta a realização dessa tarefa em períodos maiores que um ano, por exemplo, há necessidade de um recorte temporal, porém, significativo para a habitualidade $e$ as excepcionalidades de determinado clima. Utiliza-se comumente como recortes temporais nessa técnica os anos-padrão ${ }^{2}$, que são os anos mais representativos para caracterização do clima de determinado local.

A seleção de anos-padrão específicos faz-se necessária a partir de uma análise geral dos dados climáticos disponíveis. Há várias técnicas para determinação de anos-padrão de análise. Segundo (GOMES et al., 2012, p. 629),

[...] a escolha dos anos-padrão para análise rítmica tem sido orientada por diferentes critérios, ora baseados na experiência e no arbítrio dos pesquisadores, [...] ora baseados em cálculos estatísticos, [...] não havendo um único procedimento para a realização dessa tarefa.

Normalmente, os anos-padrão são escolhidos pelos totais pluviométricos sazonais e anuais, a partir de dados absolutos, diferente das temperaturas que são expressas em médias. A seleção por pluviosidade é comum, tendo em vista que esse dado é absoluto, e pode ser calculado mensal e anualmente com os totais acumulados, ou seja, mantendo a característica absoluta do dado, coadunando com o conceito não mediano do clima. Além disso, a precipitação é um elemento climático que se destaca nos climas tropicais, determinando sua sazonalidade.

A técnica de análise rítmica aplica-se a diversos estudos climáticos, incluindo estudos em conforto térmico, o que ocorre também por influência de Monteiro (2015), inserido em seu conceito de Sistema Clima Urbano, no subsistema termodinâmico. Segundo Monteiro (2015, p. 98), o conforto térmico é um dos canais de percepção humana do clima, um "filtro perceptivo bastante significativo" englobando as componentes termodinâmicas que se expressam por meio do calor, ventilação e umidade. O conforto térmico pode ser definido como "that condition of mind that expresses satisfaction with the thermal

\footnotetext{
${ }^{2}$ Pelo uso do dado absoluto, supõe-se ser raro encontrar pesquisas com períodos superiores a um ano em análise rítmica, mas o inverso já é mais frequente. Nesse caso, esses períodos menores podem receber outras denominações, até mesmo "períodos-padrão".
} 
environment and is assessed by subjective evaluation" ${ }^{\prime 3}$, conceito desenvolvido pela American Society of Heating, Refrigerating and Air Conditioning Engineers (ASHRAE, 2013, p. 3) e mais comumente aceito e utilizado entre os especialistas da área.

No entanto, o conforto térmico com aplicação de índices de conforto é raro nas pesquisas em climatologia, e mais raro ainda nos estudos envolvendo análise rítmica. Um índice térmico humano pode ser considerado como "...any parameter or indicator reported in the scientific literature that purports to represent or signify the state or significance of the thermal environment for an individual or group of individuals." ${ }^{4}$ (DE FREITAS; GRIGORIEVA, 2015, p. 110).

Como exemplo da aplicação de índices de conforto na análise rítmica, Gobo (2013) apresenta proposta de zoneamento do conforto térmico no estado do Rio Grande do Sul, com paralelo na análise rítmica de anos-padrão, mas selecionados pelo critério da pluviosidade. Gobo e Galvani (2015) propõem avaliação do padrão de conforto térmico, a partir do percentual de participação dos sistemas atmosféricos na cidade de Santa Maria (RS) em três anos-padrão, mais chuvoso, habitual e menos chuvoso, ou seja, selecionados pelos totais de precipitação.

Moura e Zanella (2012) propõem um método para a escolha de anospadrão para o estudo do conforto térmico em Fortaleza (CE), utilizando índices de conforto. As pesquisadoras utilizam medidas de tendência central e de dispersão, usando valores mensais e anuais do índice, calculados a partir dos dados de uma série histórica definida e comparam a proposta com métodos tradicionais, baseados na pluviosidade.

A presente pesquisa tem como objetivo propor um método de seleção dos anos-padrão em análise rítmica para estudos voltados ao conforto térmico, a partir de um índice de conforto térmico, e priorizando a característica absoluta do dado, por meio de análise de frequências, o que difere da proposta de Moura e Zanella (2012). Considera-se, pois, que a análise rítmica, sendo uma técnica que se coloca como alternativa ao uso de médias, não deve utilizá-las em nenhum dos elementos, inclusive no índice de conforto térmico.

A proposta é específica quanto à necessidade presente nos estudos que envolvem o conforto térmico em análise rítmica a partir de índices, exemplificada numa aplicação prática, na cidade de Palmas, Tocantins. Tal aplicação deve-se em parte aos estudos preliminares da tese de doutorado da autora, em andamento, que se propõe a contribuir com a percepção climática e o conforto térmico na referida cidade.

\section{MATERIAL E MÉTODOS}

\subsection{A ESCOLHA DO ÍNDICE}

Para seleção do índice na presente proposta, inicialmente foram identificados os mais utilizados em teses e dissertações desenvolvidas no Brasil,

\footnotetext{
${ }^{3}$ A condição da mente que expressa satisfação com o ambiente térmico, sendo avaliado de forma subjetiva. (Tradução nossa).

4 "...qualquer parâmetro ou indicador descrito na literatura científica que represente o estado ou significado do ambiente térmico para um indivíduo ou grupo de indivíduos. " (Tradução nossa).
} 
e os mais utilizados em congressos e periódicos nacionais e internacionais específicos do tema. Outro critério de seleção utilizado foi a aplicação do índice. Segundo De Freitas e Grigorieva $(2015$; 2017), praticamente todos os índices têm uma aplicação particular, um propósito específico para o mesmo. Sendo uma proposta para estudos em Climatologia Geográfica, priorizaram-se os índices utilizados em pesquisas da área. Além disso, a aplicação deveria se adequar ao uso de dados de estações climatológicas existentes.

Dentre os índices mais utilizados entre os geógrafos que estudam o clima, o Discomfort Index $\left(\mathrm{DI}_{\mathrm{T}}\right)^{5}$ criado por Thom (1959), se destaca como o mais usual. O próprio autor aplicou o índice em mais de 14 estações climatológicas americanas, resultando em mapas com isolinhas nos EUA, demonstrando que o propósito ao qual foi criado coaduna com os dados a serem utilizados nessa proposta.

Em pesquisa específica sobre a aplicação de índices de conforto térmico, Epstein e Moran (2006) recomendam o uso do $\mathrm{DI}_{\mathrm{T}}$, classificado pelos referidos autores como índice direto ${ }^{6}$, dentre outros motivos por sua fácil aplicabilidade, utilizando apenas variáveis ambientais e por sua vasta utilização há mais de 40 anos.

Já na mais recente pesquisa de comparação e classificação de 165 índices de conforto térmico, De Freitas e Grigorieva (2017) indicam o referido índice como "index based on algebraic or statistical model", ou seja, índice baseado em modelos algébricos ou estatísticos (Tradução nossa). Dentre os 37 índices nessa mesma classificação, o Discomfort Index $\left(\mathrm{DI}_{\mathrm{T}}\right)$, apesar de não ter muita abrangência (por incluir apenas duas variáveis ambientais), apresenta

${ }^{5}$ É importante ressaltar que o índice Discomfort Index de Thom (1959) obteve ao longo dos anos diversas denominações, utilizadas sem distinção, até por um mesmo pesquisador. A exemplo, Helmut Landsberg em 1972 utilizou a denominação Discomfort Index (LANDSBERG, 1972, p. 15), apresentando tanto a equação em ${ }^{\circ} \mathrm{C}$ quanto em ${ }^{\circ} \mathrm{F}$; e em 1981 utilizou Effective Temperature (LANDSBERG, 1981, p. 248), referindo-se ao mesmo índice em ${ }^{\circ} \mathrm{C}$. Ernesto Jauregui, em 1967, utilizou Discomfort Index (JAUREGUI; SOTO, 1967, p. 24) para o índice de Thom (1959) em of, e a denominação Effective Temperature para a equação em ${ }^{\circ} \mathrm{C}$. Em outras publicações, este mesmo autor utilizou a denominação Discomfort Index (JAUREGUI, 1991, p. 154), como também Effective Temperature (JAUREGUI et al., 1997, p. 167), ambas com a equação de Thom (1959) em ${ }^{\circ} \mathrm{C}$. Talvez o uso dessa dupla denominação tenha relação com a semelhança entre o Discomfort Index (DI) e o Effective Temperature (ET), pois segundo Landsberg (1972), em um intervalo considerável, os valores do DI são idênticos à ET, entre 65 e $88^{\circ} \mathrm{F}$ ET $\left(18,3\right.$ a $31,1^{\circ} \mathrm{C}$ ET). O índice Effective Temperature, citado por Landsberg (1972), foi utilizado pela ASHRAE (2013), e desenvolvido por Houghten e Yaglou (1923) apud Thom (1959). De Freitas e Grigorieva (2015; 2017), apresentam as denominações Discomfort Index ( $\mathrm{DI}_{\mathrm{T}}$ ) ou Temperature Humidity Index (THI). A versão em português do livro de Ayoade (1996, p. 65) apresenta a denominação Temperatura Efetiva (TE) em referência ao Discomfort Index de Thom (1959), e acrescenta ainda que o índice é conhecido também como Índice de Desconforto ou Índice de Temperatura-Umidade. Na realidade, em português há maior predomínio da tradução Temperatura Efetiva que a tradução Índice de Desconforto. Devido a esses conflitos, foi utilizado neste artigo apenas o nome original citado pelo próprio Thom (1959), Discomfort Index, no entanto, com a Sigla $\mathrm{DI}_{\mathrm{T}}$, adotada em catálogo desenvolvido por De Freitas e Grigorieva (2015), pela existência de mesma nomenclatura em outro índice de conforto criado posteriormente, o Discomfort Index de Ono e Kawamura (1991), desenvolvido a partir de uma modificação no Discomfort Index de Thom (1959). Referência de Houghten e Yaglou (1923) que consta em Thom (1959, p. 61): HOUGHTEN, F.C.; YAGLOU, C.P. ASHVE Research Report 673. ASHVE Transactions, v. 29, n. 361, 1923.

${ }^{6}$ Segundo os autores (EPSTEIN; MORAN, 2006, p. 391), índice direto é "an index that is based on direct measurements of environmental variables, which is used to 'simulate' heat strain", ou seja, índices diretos são aqueles baseados em medições diretas de variáveis ambientais e utilizados para "simular" tensão de calor (Tradução nossa). A classificação é baseada nos critérios do National institute for occupational safety and health (NIOSH) dos Estados Unidos (NIOSH, 1986) 
mérito conceitual e alcance razoáveis, pois tem largo intervalo de climas de aplicação, segundo a avaliação dos autores. Além disso, o índice tem facilidade de uso e foi considerado pelos autores como de alta confiabilidade de resultados, devido à larga aplicação em testes com dados empíricos de campo em diversas pesquisas no mundo. A seguir, são apresentadas as equações originais do $\mathrm{DI}_{\mathrm{T}}$ baseadas na temperatura de bulbo seco e na temperatura de bulbo úmido, a primeira em ${ }^{\circ} \mathrm{C}$ e a segunda em ${ }^{\circ} \mathrm{F}$ :

$\mathrm{DI}=0,4 \cdot\left(\mathrm{T} \_\mathrm{d}+\mathrm{T} \_\mathrm{w}\right)+4,8$

Onde: DI $\rightarrow$ Discomfort Index

$\mathrm{Td} \rightarrow$ temperatura de bulbo seco $\left({ }^{\circ} \mathrm{C}\right)$

$\mathrm{Tw} \rightarrow$ temperatura de bulbo úmido $\left({ }^{\circ} \mathrm{C}\right)$

Obs.: Temperaturas com leituras simultâneas.

Fonte: Thom $(1957 ; 1958)^{7}$ apud Landsberg (1972, p. 15).

$\mathrm{DI}=0,4 \cdot\left(T_{-} \mathrm{d}+\mathrm{T} \_\mathrm{w}\right)+15$

Onde: DI $\rightarrow$ Discomfort Index

$\mathrm{Td} \rightarrow$ temperatura de bulbo seco ( $\left.{ }^{\circ} \mathrm{F}\right)$

Tw $\rightarrow$ temperatura de bulbo úmido ( $\mathrm{O} F$ )

Obs.: Temperaturas com leituras simultâneas.

Fonte: Thom (1959, p. 59).

Outra equação foi proposta Giles et al. (1990), utilizando umidade relativa em \% em lugar da temperatura de bulbo úmido:

$\mathrm{DI}=\mathrm{Td}-0,55 \cdot(1-0,01 \cdot \mathrm{RH}) \cdot(\mathrm{Td}-14,5)$

Onde: DI $\rightarrow$ Discomfort Index

$\mathrm{Td} \rightarrow$ temperatura de bulbo seco $\left({ }^{\circ} \mathrm{C}\right)$

$\mathrm{RH} \rightarrow$ umidade relativa (\%)

Obs.: Temperaturas e umidade relativa com leituras simultâneas.

Fonte: Giles et al. (1990, p. 102).

As duas primeiras equações foram testadas ${ }^{8}$ e obtiveram coeficiente de correlação $\mathrm{R}^{2}=1$ (portanto, são idênticas). A equação adaptada por Giles et al.

\footnotetext{
${ }^{7}$ Referências que constam em Landsberg (1972, p. 36):

THOM, E. C. Cooling degree-days. Air Conditioning, Heating and Ventilating, Ref. Sec. p. 65-72, jul. 1958

THOM, E. C. A new concept for cooling degree days. Air Conditioning, Heating and Ventilating, v. 54, n. 6, p. 73-80, 1957.
} 
(1990) foi testada em comparação à original (Equação 1), e obteve forte correlação, com coeficiente $\mathrm{R}^{2}=0,98$, podendo também ser utilizada.

Foi utilizada nessa pesquisa a Equação 1, tendo em vista a disponibilidade de dados simultâneos de temperatura de bulbo seco e bulbo úmido com menor número de falhas na base de dados utilizada (INMET). No entanto, a equação proposta por Giles et al. (1990) com a umidade relativa também foi utilizada quando ocorreu ausência de dados de temperatura de bulbo úmido (em apenas 26 itens do cálculo).

Thom (1959) expressa os resultados do índice com caráter adimensional (não acrescenta o símbolo oF). No entanto, tendo em vista a utilização do índice calculado com temperaturas do ar nas duas unidades, graus Celsius e graus Fahrenheit, os resultados do índice nessa pesquisa foram representados em ${ }^{\circ} \mathrm{C}$ $\mathrm{DI}_{\mathrm{T}}$, para evitar equívocos com os resultados originados por temperaturas em graus Fahrenheit, e também por entender que o índice resultante se assemelha a uma "temperatura equivalente", coadunando com o entendimento de De Freitas e Grigorieva (2015). Dessa forma, quando o resultado do índice for 36, por exemplo, ele será representado como $36^{\circ} \mathrm{C} \mathrm{DI}$.

\subsection{A ESCOLHA DO INTERVALO DE CONFORTO}

Para a escolha do ano habitual, confortável e desconfortável para o calor e para o frio, não basta calcular o índice. É preciso identificar quais valores do índice indicam conforto e quais indicam desconforto, e a intensidade dos mesmos. Esse intervalo de referência é comumente denominado zona de conforto. Segundo o Glossário de termos para fisiologia térmica (IUPS, 2003, p. 99), zona de conforto térmico corresponde a "the range of ambient temperatures, associated with specified mean radiant temperature, humidity, and air movement, within which a human in specified clothing expresses indifference to the thermal environment for an indefinite period" ${ }^{9}$. O ideal é que o intervalo seja determinado para a região de estudo, ou seja, que tenha uma calibração. Segundo Hajdukiewicz et al. (2013, p. 291), calibração é "the adjustment of numerical and physical model input parameters to amend the agreement between the model results and corresponding experimental data" ${ }^{10}$.

Os estudos mais conhecidos voltados à calibração no Brasil têm determinado valores do índice correspondentes às respostas das pessoas de determinada localidade. As respostas podem ser de sensação térmica ou de conforto, com intervalos do índice equivalentes a esses, geralmente descritas em dois polos (de quente a frio ou de desconforto a conforto, por exemplo). Para o índice $\mathrm{DI}_{\mathrm{T}}$, o próprio Thom (1959) propôs intervalos de desconforto (por vezes, respostas fisiológicas do organismo), organizado por Giles et al. (1990) na Tabela 1 a seguir.

\footnotetext{
${ }^{8}$ Foram utilizados os dados do exemplo proposto nesse artigo, na cidade de Palmas.

${ }^{9} \mathrm{O}$ intervalo de temperatura do ambiente, associado à determinada temperatura radiante média, umidade e movimento do ar, dentro dos quais uma pessoa com determinada vestimenta expressa indiferença ao ambiente térmico por um período indefinido (Tradução nossa).

${ }^{10}$ A alteração dos parâmetros de entrada do modelo físico numérico para obter o ajuste entre os resultados do modelo e os dados experimentais correspondentes (Tradução nossa).
} 
Tabela 1 - Condições de desconforto de Thom (1959), de acordo com o Discomfort Index $\left(\mathrm{DI}_{\mathrm{T}}\right)$.

\begin{tabular}{rr} 
Condition (Condição) & DI ( ${ }^{\circ} \mathbf{C}$ ) \\
\hline $\begin{array}{r}\text { No discomfort } \\
\text { (sem desconforto) }\end{array}$ & $<21$ \\
\hline $\begin{array}{r}\text { Under 50\% of population feels discomfort } \\
\text { (menos de 50\% da população sente desconforto) }\end{array}$ & $21-24$ \\
$\begin{array}{r}\text { Over 50\% of population feels discomfort } \\
\text { (mais de 50\% da população sente desconforto) }\end{array}$ & $24-27$ \\
\hline $\begin{array}{r}\text { Most of population suffers discomfort } \\
\text { (a maioria da população sofre com o desconforto) }\end{array}$ & $27-29$ \\
\hline $\begin{array}{r}\text { Everyone feels severe stress } \\
\text { (todos sentem estresse térmico severo) }\end{array}$ & $29-32$ \\
\hline $\begin{array}{r}\text { State of medical emergency } \\
\text { (estado de emergência médica) }\end{array}$ & $>32$ \\
\hline
\end{tabular}

Fonte: Thom (1959, p. 59), organizado por Giles et al. (1990, p. 102). Tradução: Autores

No entanto, os intervalos propostos por Thom (1959) apresentam apenas um polo de desconforto, provavelmente para calor, com valores crescentes do índice, sem referência para o desconforto para frio. Além disso, esses intervalos foram aplicados nos EUA, ou seja, de clima diferente da área em estudo (Palmas). O estado do Tocantins, segundo Souza (2010, p. 166), "é marcado pela sazonalidade do regime climático, que acentua as diferenças entre a primavera-verão e o outono-inverno, principalmente no aspecto da disponibilidade hídrica na atmosfera" e ainda caracterizado por excepcionalidades, a partir de manifestação de episódios de calor, de estiagem e de pluviosidade acentuada. Do ponto de vista genético do clima, o autor destaca que o território tocantinense é influenciado por massas de ar tropicais e equatoriais. A cidade de Palmas, localizada na região central do estado do Tocantins, a cerca de $10^{\circ}$ de latitude sul e na faixa dos 200 metros de altitude (TOCANTINS, 2008), possui temperaturas altas durante todo o ano, com máximas que podem ultrapassar os $40^{\circ} \mathrm{C}$, e mínimas sempre superiores a $15^{\circ} \mathrm{C}$, segundo dados diários do Instituto Nacional de Meteorologia (INMET, 2017). Outra característica marcante é a sazonalidade da precipitação e da umidade relativa. A precipitação é frequentemente nula na estação seca, ultrapassando $80 \mathrm{~mm}$ e até $100 \mathrm{~mm}$ diários na estação úmida em eventos extremos, enquanto a umidade relativa do ar normalmente superior a $90 \%$ na estação úmida, pode ficar abaixo de $20 \%$ na estação seca (INMET, 2017). Dessa forma, fica clara a inadequação de tais intervalos à cidade de Palmas.

No Brasil, Maia e Gonçalves (2002), baseados nas condições de conforto térmico estabelecido por Fanger (1972), propuseram uma distribuição das zonas de conforto para o índice Temperatura Efetiva em função do vento (TEv) citado por Suping et al. (1992), com intervalos considerando sensação térmica e respostas fisiológicas correspondentes (Tabela 2). Como os autores apresentaram o índice TEv com forte correlação com diversos índices, dentre 
eles o $\mathrm{DI}_{\mathrm{T}}$, alguns pesquisadores têm utilizado tal distribuição para os demais índices também estudados Maia e Gonçalves (2002).

Tabela 2 - Distribuição das zonas de conforto para diferentes graus de percepção térmica e suas respostas fisiológicas, segundo Maia e Gonçalves (2002).

\begin{tabular}{ccc}
\hline TEv $\left({ }^{\circ} \mathbf{C}\right)$ & $\begin{array}{c}\text { Sensação Térmica (Tipo de } \\
\text { Tempo) }\end{array}$ & Grau de estresse fisiológico \\
\hline$<13$ & Muito Frio & Estremo estresse ao frio \\
\hline $13 \mid-16$ & Frio & Tiritar \\
\hline $16 \mid-19$ & Frio Moderado & Ligeiro resfriamento do corpo \\
\hline $19 \mid-22$ & Ligeiramente Frio & Vasoconstrição \\
\hline $22 \mid-25$ & Confortável & Neutralidade térmica \\
\hline $25 \mid-28$ & Ligeiramente Quente & Ligeiro suor; vasodilatação \\
\hline $28 \mid-31$ & Quente Moderado & Suando \\
\hline $31 \mid-34$ & Quente & Suor em profusão \\
\hline$>34$ & Muito Quente & Falha na termorregulação
\end{tabular}

Fonte: Maia e Gonçalves (2002, p. 312).

Com base nesses estudos, o Laboratório de Meteorologia Aplicada a Sistemas de Tempo Regionais do Instituto de Astronomia, Geofísica e Ciências Atmosféricas da Universidade de São Paulo (MASTER/IAG/USP), apresentou distribuição semelhante à distribuição de Maia e Gonçalves (2002), porém, incluindo maiores faixas de desconforto para o frio, apresentado na Tabela 3, a seguir. Da mesma forma que a distribuição de Maia e Gonçalves (2002), diversos pesquisadores têm utilizado tal distribuição para os demais índices estudados por eles.

Tabela 3: Distribuição das zonas de conforto para diferentes graus de percepção térmica e suas respostas fisiológicas, segundo MASTER/IAG/USP

\begin{tabular}{ccc}
\hline TE $^{\mathbf{1 1}}$ ou TEv ( $\left.{ }^{\circ} \mathbf{C}\right)$ & $\begin{array}{c}\text { Sensação Térmica (Tipo de } \\
\text { CT) }\end{array}$ & Grau de estresse fisiológico \\
\hline$<05$ & Muito Frio (MMFF) & Extremo estresse ao frio \\
\hline $05 \mid-10$ & Frio (FF) & Extremo estresse ao frio \\
\hline $10 \mid-13$ & Moderadamente Frio (MFF) & Tiritar \\
\hline $13 \mid-16$ & Ligeiramente Frio (LFF) & Resfriamento do corpo \\
\hline $16 \mid-19$ & Pouco Frio (PFF) & Ligeiro resfriamento do corpo \\
\hline $19 \mid-22$ & Ligeiramente Fresco (LF) & Vasoconstrição \\
\hline $22 \mid-25$ & Confortável (C) & Neutralidade térmica \\
\hline $25 \mid-28$ & Ligeiramente Quente (LQ) & Ligeiro suor; vasodilatação \\
\hline $28 \mid-31$ & Quente Moderado (QM) & Suando \\
\hline $31 \mid-34$ & Quente (Q) & Suor em profusão \\
\hline$>34$ & Muito Quente (MQ) & Falha na termoregulação \\
\hline
\end{tabular}

Fonte: MASTER/IAG/USP (2016, s/p).

No entanto, a distribuição de Maia e Gonçalves (2002) foi aplicada à cidade de São Paulo, enquanto a distribuição do MASTER/IAG/USP foi usada para prever as condições de conforto térmico no Sul, Sudeste e Centro-Oeste do Brasil, ou seja, novamente em climas distintos da região estudada (região

\footnotetext{
${ }^{11}$ Esse índice refere-se à Temperatura Efetiva (Effective Temperature) de Missenard (1937) apud Maia e Gonçalves (2002). Referência que consta em Maia e Gonçalves (2002, p. 314): MISSENARD, André. L'homme et le climat. Paris: Librairie Eyrolles, 1937.
} 
Norte). Além disso, os dados calculados por Maia e Gonçalves (2002) apresentam forte correlação entre os índices TEv e $\mathrm{DI}_{\mathrm{T}}$ apenas em alguns horários do dia, com coeficiente de correlação $\mathrm{R}^{2}=0,98$. Em outros horários, no entanto, o coeficiente de correlação foi $R^{2}=0,64$, ou seja, considerado baixo para a aplicação de tal distribuição.

O mais recente trabalho de calibração, a tese de doutorado de Petalas (2015), foi realizado na cidade e Fortaleza, CE. A autora definiu o limite superior e inferior de conforto baseada no percentual de votantes de acordo com a escala de sensação térmica da ASHRAE (2013), variando de calor extremo a frio extremo: a presença de "desconforto por calor" em cada medição foi constatada quando o percentual do somatório de pessoas que votaram "Calor extremo", "Muito quente", "Quente" e 50\% dos que votam "Levemente aquecido" foi superior a $31 \%$ do total dos votos coletados na medição; a presença de "desconforto por frio" em cada medição foi constatada quando o percentual do somatório de pessoas que votaram "Calor extremo", "Muito quente", "Quente", "levemente aquecido", "Neutro" e 50\% dos que votam "Levemente frio" foi inferior a $70 \%$ do total dos votos. Os limites inferior e superior do $\mathrm{DI}_{\mathrm{T}}$ identificados pela autora foram de $24,6^{\circ} \mathrm{C} \mathrm{DI}$ e $25,8^{\circ} \mathrm{C} \mathrm{DI} \mathrm{I}_{\mathrm{T}}$, respectivamente.

No entanto, identificam-se ao menos dois entraves para utilização do intervalo de conforto estabelecido por Petalas (2015). O primeiro é a faixa extremamente estreita de conforto, pouco mais de $1^{\circ} \mathrm{C} \mathrm{DI}_{\mathrm{T}}$, o que demonstra uma aplicação muito específica, válida somente para a realidade à qual foi aplicada. Além disso, a própria autora destacou que a população entrevistada apresentou uma maior tolerância ao calor, mas ainda fica a necessidade de investigação mais profunda do desconforto por frio. E o segundo entrave é, como nos outros estudos, a distância em termos climáticos entre as duas cidades. Diferente de Palmas, Fortaleza encontra-se em região litorânea, com predominância da Massa Equatorial Atlântica (MEA) e da Zona de Convergência Intertropical (ZCIT), com baixas amplitudes térmicas e pluviosidade presente em todo o ano, sendo maior de fevereiro a maio (MACIEL; ZANELLA, 2014). Além disso, Petalas (2015) frisou que os resultados podem ser estendidos apenas à região litorânea do estado do Ceará. De acordo com as características já citadas por Souza (2010), e dos dados constantes no Atlas do Tocantins (TOCANTINS, 2008) e no INMET (2017), fica novamente evidente a inadequação de tal intervalo à cidade de Palmas.

Ayoade (1996) apresenta alguns estudos com faixas de conforto do índice de Thom (1959) variando entre 20 e $21^{\circ} \mathrm{C}$ DI $\mathrm{I}_{\mathrm{T}}$ pra frio, e entre 25 e $26^{\circ} \mathrm{C}$ $\mathrm{DI}_{\mathrm{T}}$ para calor (Sul dos EUA, Europa continental, Índia, Indonésia, Malásia). No entanto, o autor considera a faixa de conforto DIT entre 18,9 e $25,6^{\circ} \mathrm{C} \mathrm{DI}$ T como uma faixa concordante entre os vários estudos em diferentes zonas climáticas. $\mathrm{Na}$ ausência de estudos que tenham calibração do $\mathrm{DI}_{\mathrm{T}}$ para a cidade em estudo e na região Norte do Brasil, utilizou-se, pois, a referência citada em Ayoade (1996), em que um $\mathrm{DI}_{\mathrm{T}}$ abaixo de $18,9^{\circ} \mathrm{C} \mathrm{DI}_{\mathrm{T}}$ configura desconforto para o frio, e acima de $25,6^{\circ} \mathrm{C} \mathrm{DI}_{\mathrm{T}}$ desconforto para o calor.

\subsection{A ESCOLHA DOS DADOS}

Antes de iniciar o tratamento dos dados climáticos para a seleção dos anos-padrão para o conforto, é necessário determinar o recorte de dados a ser 
analisado. O município de Palmas (considerando a cidade e seu entorno imediato ${ }^{12}$ ) possui seis estações meteorológicas com os dados necessários a essa pesquisa. Para o recorte amostral a ser analisado, ou seja, da estação a ser empregada, utilizou-se como critério o período de dados disponibilizados para cada estação climatológica existente. A estação convencional do INMET $^{13}$ é a mais antiga localizada na área macroparcelada da cidade, e, por esse motivo, foi utilizada para o presente estudo. A Figura 1, a seguir, mostra a localização da estação na área macroparcelada de Palmas.

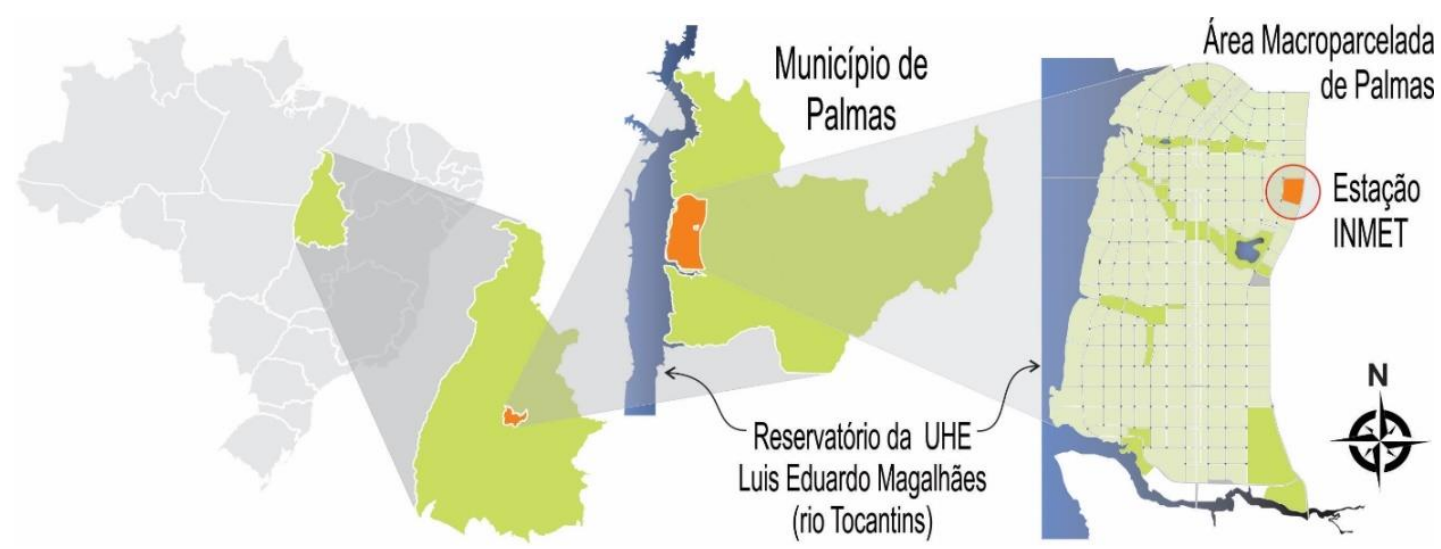

Figura 1 - Localização da Estação Convencional do INMET em Palmas (TO) Fonte: Elaborado pela autora (2017).

O INMET disponibiliza para a referida estação dados mensais, diários e horários (nos horários-padrão, 00h, $12 \mathrm{~h}$ e $18 \mathrm{~h}$ UTC, $21 \mathrm{~h}, 09 \mathrm{~h}$ e $15 \mathrm{~h}$ no horário local, respectivamente). Os dados mensais são as médias do mês inteiro. Os dados diários possuem médias (temperatura média compensada, umidade relativa e velocidade do vento) e dados absolutos (precipitação, temperatura máxima e temperatura mínima), mas não simultâneos, o que impossibilita o cálculo do índice. Os dados dos três horários-padrão fornecidos são dados instantâneos de temperatura (bulbo seco e bulbo úmido), umidade relativa, entre outros, e são os únicos absolutos e também simultâneos. Por esse motivo, foram utilizados os dados horários-padrão da estação selecionada.

Foram calculados os índices de 20 anos de dados, de 1997 a 2016. Apesar de serem disponíveis dados desde 1993, foram descartados da análise os anos 1993 a 1996, tendo em vista a grande ausência (falhas) de dados meteorológicos fornecidos pelo INMET, principalmente de ventilação, o que praticamente inviabiliza a identificação das massas de ar.

\footnotetext{
${ }^{12}$ Ressalta-se que os gradientes rurais-urbanos e intraurbanos não foram considerados, pois não são objeto da presente pesquisa.

${ }^{13}$ Código OMM 83033 (10,190 Sul, 48,30 Oeste, 280 m de altitude), inaugurada em 18 de outubro de 1993. Dados disponíveis no Banco de Dados Meteorológicos para Ensino e Pesquisa (BDMEP), no site do INMET, disponível em: <http://www.inmet.gov.br>.
} 


\subsection{PROCEDIMENTO PROPOSTO}

Por basear-se no conforto térmico, propõe-se a classificação dos anospadrão em confortáveis, desconfortáveis e habituais, além das classificações intermediárias, ou seja, habitual com alguma tendência. Ressalta-se que os anos-padrão desconfortáveis, podem apresentar características de desconforto para o frio ou para o calor, devendo ser analisados separadamente. O resultado final da análise resultará, portanto, em:

- "Ano-padrão habitual", independente se a habitualidade seja caracterizada por maior frequência de frio ou maior frequência de calor, mas a situação que mais se repete ao longo dos anos; conforto que o habitual;

"Ano-padrão confortável", ano em que houve maior frequência de

- "Ano-padrão desconfortável para o frio", quando ocorre maior frequência de desconforto para o frio que o habitual; e,

- "Ano-padrão desconfortável para o calor", quando ocorre maior frequência de desconforto para o calor que o habitual.

Após a seleção do índice térmico mais adequado ao tratamento dos dados, e seus respectivos intervalos de conforto, e de posse dos dados necessários para obtenção do mesmo, o procedimento que se propõe é o cálculo do índice com uso de uma planilha eletrônica.

A partir dos dados calculados para cada um dos horários, tem-se um banco de dados brutos do índice de conforto térmico, que é utilizado para totalização de frequências relativas. Primeiramente, são calculados os totais para cada intervalo de conforto. No caso proposto, são calculados os totais de desconforto para frio, com $\mathrm{DI}_{\mathrm{T}}$ abaixo de $18,9^{\circ} \mathrm{C} \mathrm{DI}_{\mathrm{T}}$; de conforto, com $\mathrm{DI}_{\mathrm{T}}$ entre 18,9 e $25,6^{\circ} \mathrm{C} \mathrm{DI}$; e desconforto para calor, com $\mathrm{DI}_{\mathrm{T}}$ acima de $25,6^{\circ} \mathrm{C} \mathrm{DI}_{\mathrm{T}}$, para toda a série de anos selecionada, com sua respectiva frequência relativa. Dessa forma, é possível tanto identificar se há desconforto para calor, para frio, ou para ambos, como a tendência geral da série.

Posteriormente, são calculadas as frequências relativas mês a mês para cada ano nos três intervalos, para identificar as repetições das variações mensais, fundamento da noção de "regime", segundo Monteiro (1971, p. 6). A partir desses dados, é possível calcular as diferenças entre as frequências relativas de intervalo de conforto e intervalo de desconforto para frio, assim como as diferenças entre os intervalos de conforto e desconforto para calor (sempre com a frequência de conforto subtraída da frequência de desconforto, gerando sempre valores positivos para maiores frequências de conforto, e valores negativos para maiores frequências de desconforto). Essas duas diferenças geram dois diagramas, que serão objeto de investigação do pesquisador para identificar padrões de repetição das frequências em cada mês para cada ano da série.

Com esses dois diagramas é possível perceber em cada mês, ou agrupamento de meses (trimestres, bimestres, semestres, etc.), qual o intervalo de conforto que mais se repete ao longo dos anos, assim como qual a frequência habitual. Essa forma de repetição permite identificar a habitualidade de todo o ano e, consequentemente, avaliar a classificação final proposta. 
Por ser uma análise quali-quantitativa, para facilitar a visualização das diferenças entre as frequências, utiliza-se um intervalo de cinco a seis faixas, distinguindo-as por cores ou texturas. Por exemplo, se a diferença entre o conforto e o desconforto para frio variou de $12,6 \%$ a $71,8 \%$ ao longo dos meses da série de anos selecionada, as faixas podem ser divididas em: $12 \%$ a $24 \%$; $24 \%$ a $36 \% ; 36 \%$ a $48 \%$; $48 \%$ a $60 \%$; $60 \%$ a $72 \%$, ficando a critério do pesquisador. Outro procedimento que facilita a análise dos dados é a identificação do mês de maior percentual de conforto, indicado com a letra " $\mathrm{C}$ " e o mês com maior percentual de desconforto, indicado pela letra "D", assim como o total anual, que pode ser expresso em gráfico de barras ao lado do quadro. 0 resultado final assemelha-se ao Pluviograma proposto por Schröder (1956, p. 236-243) e utilizado por Monteiro (1971, p. 5), motivo pelo qual foi denominado de "Confortograma", porém, diferente do Pluviograma, tem-se dois Confortogramas, um para o frio e outro para o calor.

Os Confortogramas não são os únicos, mas os principais instrumentos de análise nessa proposta para a classificação final dos anos-padrão com base no conforto térmico, pois auxiliam na identificação das variações mensais em sucessivos anos.

\section{RESULTADOS E DISCUSSÃO}

\subsection{RESULTADOS}

Seguindo o procedimento proposto, foram calculados os índices para os horários de 00, 12 e 18 UTC da série de 1997 a 2016 da estação meteorológica selecionada, totalizando 23651 índices calculados, sendo posteriormente totalizados os percentuais de frequência relativa nos três intervalos de conforto do $\mathrm{DI}_{\mathrm{T}}$, resultando no Quadro 1.

Quadro 1: Frequências relativas para cada intervalo de conforto do $\mathrm{DI}_{\mathrm{T}}$, entre $1997 \mathrm{e}$ 2016 em Palmas (TO). Fonte: INMET (2017), Org. SILVA, L. F. G. (2017)

\begin{tabular}{|c|c|c|}
\hline $\begin{array}{c}\text { DESCONFORTO PARA O } \\
\text { FRIO } \\
\leq 18,9^{\circ} \mathrm{C} \mathrm{DI}_{\mathrm{T}}\end{array}$ & $\begin{array}{c}\text { CONFORTO } \\
18,9^{\circ} \mathrm{C} \mathrm{DI}_{\mathrm{T}}<\mathrm{X} \leq 25,6^{\circ} \mathrm{C} \mathrm{DI}_{\mathrm{T}}\end{array}$ & $\begin{array}{c}\text { DESCONFORTO PARA O } \\
\text { CALOR } \\
>25,6^{\circ} \mathrm{C} \mathrm{DI}_{\mathrm{T}}\end{array}$ \\
\hline $0,000 \%$ & $58,203 \%$ & $41,797 \%$ \\
\hline
\end{tabular}

O menor valor registrado do índice foi $19,24^{\circ} \mathrm{C} \mathrm{DI}_{\mathrm{T}}$, em 8 de julho de 1999, às 00 UTC, e o maior registrado foi $33,19^{\circ} \mathrm{C} \mathrm{DI}_{\mathrm{T}}$ em 12 de maio de 2015, às 18 UTC. A maior frequência registrada foi de conforto, com 16,3\% a mais que o desconforto para calor. Verifica-se que na cidade de Palmas, não foram registrados índices $\mathrm{DI}_{\mathrm{T}}$ com desconforto para o frio, dispensando a necessidade do Confortograma para o frio. A análise deverá avaliar, portanto, apenas as diferenças entre os intervalos de conforto e de desconforto para calor.

Em seguida, foram calculadas as frequências de conforto e desconforto para calor para cada mês em cada ano da série, e calculadas as diferenças entre as frequências desses dois intervalos (frequência de conforto menos a frequência de desconforto para calor). A maior diferença positiva mensal registrada, ou seja, mais conforto que desconforto para o calor, foi de $57,1 \%$ no mês de fevereiro nos anos de 2007 e 2014, enquanto a maior diferença 
negativa mensal, ou seja, mais desconforto para calor que conforto, foi de $56,3 \%$ também no mês de fevereiro, porém de 2016. As diferenças entre as frequências relativas do intervalo de conforto e desconforto para o calor, por mês e ano da série selecionada, foram utilizadas para elaboração do Confortograma para o calor (Quadro 2), onde também foram totalizadas as diferenças anuais (total da frequência de conforto no ano menos total da frequência de desconforto para calor no ano).

Quadro 2: Confortograma para o calor em Palmas (TO) (1997-2016) Fonte: INMET (2017), Org. SILVA, L. F. G. (2017)

\begin{tabular}{|c|c|c|c|c|c|c|c|c|c|c|c|c|c|c|}
\hline \multirow{2}{*}{ ANO } & \multicolumn{12}{|c|}{ MESES } & \multirow{2}{*}{\multicolumn{2}{|c|}{$\begin{array}{c}\text { DIFERENÇA TOTAL } \\
\text { ANUAL }\end{array}$}} \\
\hline & J & $F$ & $M$ & A & $M$ & J & J & A & $S$ & 0 & $\mathbf{N}$ & D & & \\
\hline 1997 & & & C & & & & & & & $\mathrm{D}$ & & & $25,7 \%$ & \\
\hline 1998 & & & & $D$ & & & C & & & & & & $8,8 \%$ & \\
\hline 1999 & & & C & & & & & & $\mathrm{D}$ & & & & $29,8 \%$ & \\
\hline 2000 & & C & & & & & & & $\bar{D}$ & & & & $33,0 \%$ & \\
\hline 2001 & & C & & & & & & & $\bar{D}$ & & & & $30,8 \%$ & \\
\hline 2002 & C & & & & & & & & & & D & & $16,2 \%$ & \\
\hline 2003 & & & C & & & & & & & $\mathrm{D}$ & & & $18,7 \%$ & \\
\hline 2004 & & C & & & & & & & $\mathrm{D}$ & & & & $16,7 \%$ & \\
\hline 2005 & & & & & & & C & & & D & & & $9,3 \%$ & \\
\hline 2006 & & & C & & & & & & & D & & & $19,3 \%$ & \\
\hline 2007 & & C & & & & & & & & D & & & $12,7 \%$ & \\
\hline 2008 & & & C & & & & & & & D & & & $23,1 \%$ & \\
\hline 2009 & & C & & & & & & & $\mathrm{D}$ & & & & $19,1 \%$ & \\
\hline 2010 & & & & D & & & C & & & & & & $-0,3 \%$ & \\
\hline 2011 & & C & & & & & & & $\mathrm{D}$ & & & & $28,4 \%$ & \\
\hline 2012 & C & & & & & & & & & D & & & $17,7 \%$ & \\
\hline 2013 & C & & & & & & & & D & & & & $11,8 \%$ & \\
\hline 2014 & & C & & & & & & & $D$ & & & & $21,5 \%$ & \\
\hline 2015 & & & & & & & C & & & D & & & $2,1 \%$ & \\
\hline 2016 & & D & & & & & C & & & & & & $-13,4 \%$ & \\
\hline
\end{tabular}

Legenda:

\begin{tabular}{rcc}
$\square-60 \%$ & $\vdash$ & $-40 \%$ \\
$-40 \%$ & $\vdash$ & $-20 \%$ \\
$-20 \%$ & $\vdash$ & $0 \%$ \\
\hline $0 \%$ & $\vdash$ & $20 \%$ \\
$20 \%$ & $\vdash$ & $40 \%$ \\
$40 \%$ & $\vdash$ & $60 \%$
\end{tabular}

C Mês com maior percentual de conforto no ano

D Mês com maior percentual de desconforto para o calor no ano

\subsection{ANÁLISE E DISCUSSÃO}

De posse do Confortograma, é possível identificar uma habitualidade para a cidade de Palmas. Verifica-se que, de setembro a novembro estão os meses com altos e médios percentuais do $\mathrm{DI}_{\mathrm{T}}$ de desconforto para calor, sendo que os maiores estão em setembro e outubro. Em dezembro, há predominância de baixos e médios percentuais de conforto. De janeiro a março, estão os mais altos percentuais do $\mathrm{DI}_{\mathrm{T}}$ de conforto. Em abril e maio, há predominância de baixos e médios percentuais de conforto, e em alguns momentos percentuais baixos de desconforto para calor. De junho a agosto, há predominância de 
médios percentuais de conforto, principalmente no mês de julho. Os anos mais representativos dessa habitualidade são 2008 e 2009. Esses dois anos apresentam o mês mais confortável no verão, contando com a ajuda do tempo chuvoso, e o mais desconfortável na transição inverno-primavera, no final do período seco, o que reafirma sua habitualidade. Outros anos que também apresentam essa habitualidade, mas com alguma tendência à confortável ou desconfortável para o calor são: 2002, 2003, 2006 e 2014, habituais com tendência a confortáveis; e 1999 e 2004, habituais com tendência a desconfortáveis. Verificou-se que, na cidade de Palmas, é habitual que haja algum grau de desconforto, especialmente em determinadas épocas do ano, pois isso é o que mais se repete.

Para identificar os anos não habituais, ou seja, mais desconfortáveis para o calor e mais confortáveis que o habitual, considerou-se o não atendimento às características descritas no parágrafo anterior. No caso de classificação de anospadrão mais desconfortáveis para o calor, verificou-se:

- $\quad$ Se existiam percentuais de desconforto para o calor em períodos típicos de maior percentual de conforto;

- $\quad$ Ausência de alguma habitualidade para o conforto (percentuais mais baixos que o habitual, ou nulos); e

- Presença de alguma anomalia positiva para desconforto (percentuais mais altos que o habitual).

Foram considerados anos desconfortáveis para o calor os anos 1998, 2005, 2007, 2010, 2012, 2013, 2015 e 2016. O ano 1998, pois apresenta desconforto maior em período não típico, no mês de abril, ausência de percentuais altos de conforto em período típico, e mantém o desconforto, porém médio, em setembro e outubro. O ano 2005, pois apresenta desconforto médio em período não típico, fevereiro e abril, ausência de percentuais altos de conforto em período típico, e mantém o desconforto de setembro a novembro. 0 ano 2007, pois apresenta desconforto maior em período não típico, no mês de março, e mantém o desconforto em setembro e novembro. O ano 2010, pois apresenta desconforto maior em período não típico, fevereiro a maio, e mantém o desconforto em setembro e outubro. O ano 2012, pois apresenta desconforto maior em período não típico, no mês de dezembro, e mantém o desconforto em setembro e outubro, sendo outubro com desconforto alto. O ano 2013, pois apresenta desconforto médio em período não típico, em abril, ausência de percentuais altos de conforto em período típico, e mantém o desconforto em setembro e outubro. O ano 2015, pois apresenta percentuais altos de desconforto tanto em período típico, setembro a novembro, como em dezembro, período não típico, ausência de percentuais altos de conforto em período típico. $\mathrm{O}$ ano 2016, pois apresenta percentuais altos de desconforto tanto em período típico, setembro a novembro, como de fevereiro a maio, quando seria típico mais conforto.

Para identificar os anos-padrão mais confortáveis que o habitual, verificou-se:

Se existiam percentuais de conforto em períodos típicos de maior percentual de desconforto para calor; 
- $\quad$ Ausência de alguma habitualidade para o desconforto (percentuais mais baixos que o habitual, ou nulos); e

- $\quad$ Presença de alguma anomalia positiva para conforto (percentuais mais altos que o habitual).

Foram considerados anos confortáveis os anos 1997, 2000, 2001 e 2011. O ano 1997, por apresentar meses de percentual alto de conforto em períodos habituais e também não habituais, como maio e julho, e também, apesar de manter percentuais de desconforto de setembro a novembro, por apresentar percentuais mais baixos. O ano 2000, por não possuir nenhum mês com percentual de desconforto e manter percentuais altos de conforto em período típico. O ano 2001, por não possuir nenhum mês com percentual de desconforto e manter percentuais altos de conforto em período típico. $O$ ano 2011, por não possuir nenhum mês com percentual de desconforto e manter percentuais altos de conforto em período típico e também por apresentar percentual de conforto em período não típico, em novembro.

A classificação final dos anos-padrão para estudos em conforto térmico, baseados no Confortograma, encontra-se no Quadro 3. Ao total, foram classificados quatro anos confortáveis, oito desconfortáveis para o calor e oito habituais (incluindo os anos habituais que apresentaram alguma tendência). Uma característica percebida na cidade de Palmas, foi o número elevado de anos desconfortáveis para o calor em relação aos confortáveis e aos habituais. Normalmente, as excepcionalidades são menos frequentes que a habitualidade, o que demonstra que o número considerável de anos desconfortáveis para o calor também possa representar certa habitualidade para Palmas.

Quadro 3: Classificação final dos anos-padrão no Confortograma para o calor em Palmas (TO) (1997-2016). Fonte: INMET (2017), Org. SILVA, L. F. G. (2017)

\begin{tabular}{|c|c|c|c|c|c|c|c|c|c|c|c|c|c|}
\hline \multirow{2}{*}{ ANO } & \multicolumn{12}{|c|}{ MESES } & \multirow{2}{*}{ CLASSIFICAÇÃO ANOS-PADRÃO } \\
\hline & $\mathbf{J}$ & $\mathbf{F}$ & $\mathbf{M}$ & A & $\mathbf{M}$ & $\mathbf{J}$ & $\mathbf{J}$ & A & $\mathbf{S}$ & $\mathbf{0}$ & $\mathbf{N}$ & D & \\
\hline 1997 & & & $\mathrm{C}$ & & & & & & & $\mathrm{D}$ & & & CONFORTÁVEL \\
\hline 1998 & & & & $\mathrm{D}$ & & & $\mathrm{C}$ & & & & & & DESCONFORTÁVEL PARA O CALOR \\
\hline 1999 & & & $\mathrm{C}$ & & & & & & $\mathrm{D}$ & & & & HABITUAL A DESCONFORTÁVEL PARA O CALOR \\
\hline 2000 & & $\mathrm{C}$ & & & & & & & $\mathrm{D}$ & & & & CONFORTÁVEL \\
\hline 2001 & & $\mathrm{C}$ & & & & & & & D & & & & CONFORTÁVEL \\
\hline 2002 & $\mathrm{C}$ & & & & & & & & & & $\mathrm{D}$ & & HABITUAL A CONFORTÁVEL \\
\hline 2003 & & & $\mathrm{C}$ & & & & & & & $\mathrm{D}$ & & & HABITUAL A CONFORTÁVEL \\
\hline 2004 & & $\mathrm{C}$ & & & & & & & D & & & & HABITUAL A DESCONFORTÁVEL PARA O CALOR \\
\hline 2005 & & & & & & & $\mathrm{C}$ & & & $\mathrm{D}$ & & & DESCONFORTÁVEL PARA O CALOR \\
\hline 2006 & & & $\mathrm{C}$ & & & & & & & $\mathrm{D}$ & & & HABITUAL A CONFORTÁVEL \\
\hline 2007 & & C & & & & & & & & D & & & DESCONFORTÁVEL PARA O CALOR \\
\hline 2008 & & & $\mathrm{C}$ & & & & & & & D & & & HABITUAL \\
\hline 2009 & & $\mathrm{C}$ & & & & & & & D & & & & HABITUAL \\
\hline 2010 & & & & $\mathrm{D}$ & & & C & & & & & & DESCONFORTÁVEL PARA O CALOR \\
\hline 2011 & & C & & & & & & & $\mathrm{D}$ & & & & CONFORTÁVEL \\
\hline 2012 & C & & & & & & & & & D & & & DESCONFORTÁVEL PARA O CALOR \\
\hline 2013 & $\mathrm{C}$ & & & & & & & & $\mathrm{D}$ & & & & DESCONFORTÁVEL PARA O CALOR \\
\hline 2014 & & $\mathrm{C}$ & & & & & & & D & & & & HABITUAL A CONFORTÁVEL \\
\hline 2015 & & & & & & & C & & & D & & & DESCONFORTÁVEL PARA O CALOR \\
\hline 2016 & & D & & & & & C & & & & & & DESCONFORTÁVEL PARA O CALOR \\
\hline
\end{tabular}




\section{CONCLUSÕES}

A metodologia proposta apresentou-se eficiente dentro dos parâmetros apresentados e considerando a necessidade de correlacionar a análise rítmica aos estudos em conforto térmico, especificamente com índices. A partir dessa metodologia, é possível realizar uma análise rítmica que considere o ritmo do comportamento do conforto térmico, com suas formas habitual e excepcional, ao longo dos anos em uma determinada localidade, demonstrando a interferência de todos os elementos climáticos sobre o mesmo, e não só da pluviosidade.

A metodologia foi realizada com o índice $\mathrm{DI}_{\mathrm{T}}$, mas é adequada para outros índices de conforto térmico, caso o pesquisador identifique como mais adequado para a área de estudo. No entanto, o mesmo não ocorre para os intervalos de conforto. O intervalo do $\mathrm{DI}_{\mathrm{T}}$ utilizado apresenta apenas três faixas, mas há intervalos maiores, como o utilizado por Maia e Gonçalves (2002) e do MASTER/IAG/USP (2016), mostrado nas Tabelas 2 e 3, que apresentam diversas faixas. Nesse caso, recomenda-se que os intervalos sejam agrupados, de forma a simplificar o cálculo. No exemplo do MASTER/IAG/USP (2016), seriam agrupadas as faixas de "Ligeiramente Fresco" a "Muito Frio", e de "Ligeiramente Quente" a "Muito Quente", tendo em vista que a produção de muitos Confortogramas pode confundir o pesquisador, ao invés de facilitar a seleção dos anos-padrão.

A escolha da(s) estação(ões) para elaboração do Confortograma, demonstrou também a necessidade de um extremo cuidado no universo de dados a ser analisado, considerando os dados necessários ao cálculo de um determinado índice, a série de anos disponível e a sua representatividade.

O estudo de caso apresentado para a cidade de Palmas, apresentou apenas o Confortograma para o calor, mas no caso de cidade com ambas as situações, para calor e frio, os Confortogramas devem ser analisados em conjunto, tanto para a avaliação final dos anos-padrão desconfortáveis para o calor, desconfortáveis para o frio, e principalmente, confortáveis e habituais, que estarão presentes nos dois Confortogramas.

\section{REFERÊNCIAS}

AMERICAN SOCIETY OF HEATING, REFRIGERATING AND AIR CONDITIONING ENGINEERS (ASHRAE). ANSI/ASHRAE Standard 55: Thermal environmental conditions for human occupancy. Atlanta, GA, 2013.

AYOADE, Johnson Olaniyi. Introdução à climatologia para os trópicos. 4. ed. Tradução Maria Juraci Zani dos Santos. Rio de Janeiro: Bertrand Brasil, 1996.

DE FREITAS, Christopher R.; GRIGORIEVA, Elena A. A comprehensive catalogue and classification of human thermal climate indices. International Journal of Biometeorology, v. 59, n. 1, p. 109-120, jan. 2015.

. A comparison and appraisal of a comprehensive range of human thermal climate indices. International Journal of Biometeorology, v. 61, n. 3, p. 487-512, mar. 2017.

EPSTEIN, Yoram; MORAN, Daniel S. Thermal comfort and the heat stress indices. Industrial Health, v. 44, n. 3, p. 388-398, 2006. 
GILES, Brian D.; BALAFOUTIS, Christos; MAHERAS, Panyotis. Too hot for comfort: The heatwaves in Greece in 1987 and 1988. International Journal of Biometeorology, v. 34, n. 2, p. 98-104, jun. 1990.

GOBO, João Paulo Assis. Regionalização climática do Rio Grande do Sul com base no zoneamento do conforto térmico humano. 2013. 184 p. Dissertação (Mestrado em Geografia Física) - Universidade de São Paulo, São Paulo, SP, 2013.

GOBO, João Paulo Assis; GALVANI, Emerson. Inserção do estudo da dinâmica atmosférica regional na análise dos padrões de conforto térmico humano no Rio Grande do Sul: estudo de caso em Santa Maria-RS. Geousp - Espaço e Tempo, v. 19 , n. 3, p. 564-584, set./dez. 2015.

GOMES, Luam Patrique de Oliveira; SILVA, Aion Angelu Ferraz; SOUZA, Lucas Barbosa e. Notas sobre a seleção de anos-padrão para o estudo da gênese e da dinâmica climática no estado do Tocantins: aspectos metodológicos a partir da inclusão do critério espacial. Revista Geonorte, v. 1, n. 5, p. 628-641, 2012

HAJDUKIEWICZ, Magdalena; GERON, Marco; KEANE, Marcus M. Formal calibration methodology for CFD models of naturally ventilated indoor environments. Building and Environment, v. 59, p. 290-302, jan. 2013.

INSTITUTO NACIONAL DE METEOROLOGIA (INMET). Banco de Dados Meteorológicos para Ensino e Pesquisa (BDMEP). Disponível em: <http://www.inmet.gov.br/ portal/index.php?r=bdmep/bdmep>. Acesso em: 28 jan. 2017.

INTERNATIONAL UNION OF PHYSIOLOGICAL SCIENCES (IUPS). Glossary of terms for thermal physiology. Journal of Thermal Biology, 3. ed., v. 28, n. 1, p. 75-106, jan. 2003.

JAUREGUI, Ernesto; CERVANTES, Juan; TEJEDA, Adalberto. Bioclimatic conditions in Mexico City: an assessment. International Journal of Biometeorology, v. 40, n. 3, p. 166-177, maio 1997.

JAUREGUI, Ernesto; SOTO, C. Wet-Bulb Temperature and Discomfort Index Areal Distribution in Mexico. International Journal of Biometeorology, v. 11, $\mathrm{n}$. 1, p. 21-28, mar. 1967.

JAUREGUI, Ernesto. The human climate of tropical cities: an overview. International Journal of Biometeorology, v. 35, n. 3, p. 151-160, set. 1991.

LANDSBERG, Helmut Erich. The assessment of human bioclimate: a limited review of physical parameters. World Meteorological Organization, Technical Note n. 123. Genebra, Suíca: WMO, 1972.

. The Urban Climate. International Geophysics series, v. 28. Nova Iorque: Academic Press, 1972.

MACIEL, Leandro Leite da Silva; ZANELLA, Maria Elisa. Clima urbano e conforto térmico em Fortaleza - os terminais de integração. In: SIMPÓSIO BRASILEIRO DE CLIMATOlOGIA GEOGRÁFICA, 10., out. 2014, Curitiba. Anais... Curitiba: ABClima, 2014. p. 367-379.

MAIA, João André; GONÇALVES, Fábio Luiz Teixeira. Uma análise do conforto térmico e suas relações meteorotrópicas na cidade de São Paulo - parte 1. In: 
CONGRESSO BRASILEIRO DE METEOROLOGIA, 12., 2002, Foz de Iguaçu. Anais... Foz de Iguaçu: SBMET, 2002. p. 305-314.

METEOROLOGIA APLICADA A SISTEMAS DE TEMPO REGIONAIS. Astronomia, Geofísica e Ciências Atmosféricas. Universidade de São Paulo (MASTER/IAG/USP). Distribuição das zonas de conforto para diferentes graus de percepção térmica e suas respostas fisiológicas. Disponível em: <http://www.masterantiga.iag.usp.br/conforto/

previsao.php?ant $=\& v a r=$ temp_efet $\& \mathrm{t}=2 \& \mathrm{gr}=2 \& p r e f=2 \mathrm{~g} \& \mathrm{inic}=00>$. Acesso em: 30 out. 2016.

MONTEIRO, Carlos Augusto de Figueiredo. Notas para o Estudo do Clima do Centro-oeste Brasileiro. Revista Brasileira de Geografia, v. 13, n. 1, p. 3-46, jan./mar. 1951.

Da necessidade de um caráter genético à classificação climática: algumas considerações metodológicas a propósito do estudo do Brasil Meridional. Revista Geográfica, Rio de Janeiro: Instituto Pan-Americano de Geografia e História, v. 31, n. 57, p. 29-44, 1962.

Sôbre a análise geográfica de seqüências de cartas de tempo: pequeno ensaio metodológico sôbre o estudo do clima no escôpo da geografia. Revista Geográfica, Rio de Janeiro: Instituto Pan-Americano de Geografia e História, v. 32 , n. 58, p. 169-179, 1963.

Sobre um índice de participação das massas de ar e suas possibilidades de aplicação à classificação climática. Revista Geográfica, Rio de Janeiro: Instituto Pan-Americano de Geografia e História, v. 33, n. 61, p. 59-69, 1964.

A frente polar atlântica e as chuvas de inverno na fachada sul-oriental do Brasil: contribuição metodológica à análise rítmica dos tipos de tempo no Brasil. São Paulo: Universidade de São Paulo/Instituto de Geografia, 1969.

Análise rítmica em climatologia: problemas da atualidade climática em São Paulo e achegas para um programa de trabalho. São Paulo: Universidade de São Paulo/Instituto de Geografia, 1971.

. A climatologia geográfica no Brasil e a proposta de um novo paradigma. In: MONTEIRO, Carlos Augusto de Figueiredo (Org.); SANT'ANNA NETO, João Lima; MENDONÇA, Francisco de Assis; ZAVATTINI, João Afonso. A construção da climatologia geográfica no Brasil. Campinas, SP: Editora Alínea, 2015. p. 61153.

MOURA, Marcelo de Oliveira; ZANELLA, Maria Elisa. Escolha de anos-padrão para o estudo do conforto térmico em Fortaleza, CE: verificação de critérios. Revista Geonorte, v. 1, n. 5, edição Especial 2, p. 547-560, 2012.

NATIONAL INSTITUTE FOR OCCUPATIONAL SAFETY AND HEALTH (NIOSH). Criteria for a recommended standard: occupational exposure to hot environment. Publication n. 86-113. Washington DC.: NIOSH, 1986.

ONO, Hye-Suok Park; KAWAMURA, Takeshi. Sensible climates in monsoon Asia. International Journal of Biometeorology, v. 35, n. 1, p. 39-47, mar. 1991.

PETALAS, Kety Vasconcelos. Estudo da sensação térmica e definição de limites de conforto para espaços abertos na cidade de Fortaleza, CE. 2015. 272 p. Tese 
(Doutorado em Engenharia Civil) - Universidade Federal do Ceará, Fortaleza, 2015.

RIBEIRO, Antonio Giacomini. As escalas do clima. Boletim de Geografia Teorética, v. 23, n. 45-46, p. 288-294, 1993.

SCHRÖDER, Rudolf. Distribuição e curso anual das precipitações no Estado de São Paulo. Bragantia, Instituto Agronômico de Campinas, v. 15, n. 18, p. 193249, ago. 1956.

SORRE, Maximillien. Le Climat. In: . Les fondements de la Géographie Humaine. Tomo I: Les fondements biologiques: Essai d'une écologie de I'homme. Livro I: Le climat et I'homme. 3. ed. Paris: Librairie Armand Colin, 1951. cap. 1, p. 13-43.

SOUZA, Lucas Barbosa e. Novas cidades, velhas querelas: episódios pluviais e seus impactos na área urbana de Palmas (TO), primavera-verão 2009/2010. Mercator, Fortaleza, CE, v. 9, número especial 1, p. 165-177, dez. 2010.

SUPING, Zhang; GUANGLIN, Meng; YANWEN, Wang; JI, Li. Study of the relationships between weather conditions and the marathon race, and of meteorotropic effects on distance runners. International Journal of Biometeorology, v. 36, n. 2, p. 63-68, jun. 1992.

THOM, Earl Crabill. The Discomfort Index. Weatherwise, v. 12, n. 2, p. 57-61, abr. 1959.

TOCANTINS (Estado). Secretaria de Planejamento e Meio Ambiente. Diretoria de Zoneamento Ecológico-Econômico. Atlas do Tocantins: subsídios ao planejamento da gestão territorial. Palmas: SEPLAN, 2008 